\title{
A fatigue damage model for seismic response of $\mathrm{RC}$ structures
}

\author{
R. Perera ${ }^{\mathrm{a}, *}$, A. Carnicero ${ }^{\mathrm{b}}$, E. Alarcon ${ }^{\mathrm{a}}, \mathrm{S}$. Gomez ${ }^{\mathrm{a}}$ \\ ${ }^{a}$ ETS Ingenieros Industriales, Technical University of Madrid, 28006 Madrid, Spain \\ ${ }^{\mathrm{b}}$ Universidad Pontificia Comillas, 28015 Madrid, Spain
}

\begin{abstract}
Numerous damage models have been developed in order to analyze seismic behavior. Among the different possibilities existing in the literature, it is very clear that models developed along the lines of continuum damage mechanics are more consistent with the definition of damage as a phenomenon with mechanical consequences because they include explicitly the coupling between damage and mechanical behavior. On the other hand, for seismic processes, phenomena such as low cycle fatigue may have a pronounced effect on the overall behavior of the frames and, therefore, its consideration turns out to be very important. However, most of existing models evaluate the damage only as a function of the maximum amplitude of cyclic deformation without considering the number of cycles. In this paper, a generalization of the simplified model proposed by Cipollina et al. [Cipollina A, López-Hinojosa A, Flórez-López J. Comput Struct 1995;54:1113-26] is made in order to include the low cycle fatigue. Such a model employs in its formulation irreversible thermodynamics and internal state variable theory.
\end{abstract}

Keywords: Continuum damage mechanics; Low cycle fatigue; Reinforced concrete

\section{Introduction}

In recent years, many studies have been developed to evaluate the seismic behavior using the damage index concept. Damage indices aim to provide a means for quantifying structural degradation sustained under earthquake loading and therefore define a structural safety measure. These indices are of extensive use in characterization of vulnerability curves which relate the damage quantification to a measure of the ground motion severity (the peak acceleration, for instance). Hence, the evaluation and calibration of damage indices are important in order to evaluate the safety of new and existing structures and also in establishing a framework for seismic retrofitting decision making of structures.
Obviously, characterization of structural damage is a subjective matter, so the main problem is its quantification. In fact, there are many proposed models in the literature to evaluate it. Most of these are based essentially on a post-earthquake evaluation. These types of models include the effect of damage only implicitly and only to a certain degree; damage is not considered as an internal variable, and there is no coupling between the nonlinear computation of the inelastic response and the computation of the evolution of damage. However, the degree of damage certainly affects mechanical behavior.

In this paper, the proposed model is based on the notions and principles of continuum damage mechanics and, therefore, on thermodynamics of irreversible processes, internal state variable theories and physical considerations. A scalar damage variable is suitable for characterizing isotropic damage processes. Through the damage index, the effective stress concept and the strain equivalence principle, a mechanical representation of damage is obtained. The models developed along the 
lines of continuum damage mechanics explicitly include the coupling between damage and mechanical behavior and are consistent with the definition of damage as a phenomenon with mechanical consequences.

In order to adapt the continuum problem to frame analysis, a generalization of the concepts used in "lumped plasticity models" is employed in order to include the dissipative effects produced by structural damage as proposed by Cipollina et al. [1] and FlórezLópez [2]. According to this model, damage effects are assumed to be concentrated at the ends of the member. This formulation can be considered as simplified damage mechanics for frames and allows its implementation in finite element programs. By incorporating some notions and concepts of continuum damage mechanics to the simplified model, the constitutive equations are obtained.

An important aspect for evaluating damage is related to the consideration of peak values as damage parameters. Examples of such are peak displacements or deformations in the positive or negative sense or cumulative measures of response quantities, such as total dissipated energy, etc., or a combination of both. The choice of one or another will depend on the loading type and the material. So, in monotonic loading, peak values are suitable for quantifying the damage evolution. However, in cyclic loading when only the first type of damage parameters are considered, the computation considers cyclic loading as equivalent to monotonic, since damage parameters have the same values in both cases. In this case, it would be necessary to consider parameters which allow representation of the fatigue effects.

In previous coupled simplified models [1,2], the damage is only a function of the maximum amplitude of cyclic deformation experienced by the member; therefore, cyclic loading effects cannot be represented. This constitutes an important limitation of the model as it is well known that, for example, during strong earthquakes connections may fail due to low cycle fatigue produced as a result of cyclic inelastic rotations. So, it is necessary to modify the model in order to consider such effects.

It is the purpose of this paper to present a formulation that allows us to take account of the strength degradation produced, not only by the peak values, but also by the cumulative effects. This type of formulation is attractive because it can handle in a unified manner monotonic loading, low cycle and high cycle fatigue. To achieve this, the concepts of continuum damage mechanics are used.

To include the cyclic loading effects, various approaches have been proposed in the literature to formulate the fatigue damage law. However, in these models, classical concepts of continuum damage mechanics, such as the damage energy release rate [3] or the damage surface, are changed [4] or left [5-7] for a loading-unloading irreversibility concept, respectively.
The alternative proposed here is related to the dissipative potential. Using a coupled simplified model, the potential based on Griffith criterion proposed in Refs. [1,2] is reformulated in order to include the cumulative effects in the damage evolution whilst keeping unchanged the concept of damage energy release rate usually employed. Compared with other models, the usual yield and damage criteria are kept and all the new parameters, introduced to evaluate the fatigue damage, have physical meaning so they can be measured or identified physically.

After a short description of the simplified model in Section 2, a damage mechanism is proposed in the following section to describe the fatigue damage. Experimental results are used as a comparison in order to determine the validity of the model.

\section{Lumped plastic-damageable model}

Physically, degradation of material properties is related to the initiation, growth and coalescence of microcracks. Due to the existence of microcracks, the flexibility of a material increases.

According to the strain equivalence principle [8], for an elastic-damage case, if $E$ denotes the undamaged stiffness, the unloading stiffness of the damaged material is defined by $E(1-d)$, where $d$ is an isotropic damage variable. If it is assumed that all microcracks close upon unloading, no permanent deformation remains after the complete unloading. However, the truly reversible elastic strain $\varepsilon^{\mathrm{e}}$ is obtained by

$\varepsilon^{\mathrm{e}}=\frac{\sigma}{E}$.

The rest of the strain is actually the inelastic strain $\varepsilon^{\mathrm{d}}$ due to the microcrack opening during the loading process and brings in the effect of the degradation of elastic properties $[4,9]$ :

$\varepsilon^{\mathrm{d}}=\frac{\sigma}{E(1-d)}-\frac{\sigma}{E}=\frac{\sigma d}{E(1-d)}$.

This result is consistent with the principal phenomena observed in the response of concrete under uniaxial loading [10].

Eq. (2) can be specified for the particular case of a truss member. Denoting by $N, \delta^{\mathrm{d}}$ and $d_{\mathrm{a}}$ the axial force, the damage elongation and the axial damage, respectively, it follows that

$\delta^{\mathrm{d}}=\frac{N L}{E A} \frac{d_{\mathrm{a}}}{\left(1-d_{\mathrm{a}}\right)}$.

This result constitutes the basis of the model proposed by Flórez-López [2]. According to this model, using an approach similar to that employed in lumped plasticity 
models and in order to include the damage effects, it is assumed that not only the plastic deformations but also the damage deformations are concentrated at the hinges, i.e., all the dissipative phenomena occur at the hinges.

This assumption allows the simulation of the degradation of frames using a simplified model. For this model, a frame member is idealized by an elastic element considering the dissipative effects lumped at its ends. More details about this mechanical model can be found in Ref. [11].

The stress distribution for each element is described by a three component vector $q$ that collects the bending moments at the two ends and the axial force. The corresponding kinematic variable $u$ define the deformed shape of the element excluding the rigid body motion (Fig. 1).

From Eq. (3), a similar relationship is postulated for flexural effects.

$\theta_{\mathrm{i}}^{\mathrm{d}}=\frac{d_{\mathrm{i}}}{1-d_{\mathrm{i}}} \frac{L}{4 E I} M_{\mathrm{i}}$,

$\theta_{\mathrm{j}}^{\mathrm{d}}=\frac{d_{\mathrm{j}}}{1-d_{\mathrm{j}}} \frac{L}{4 E I} M_{\mathrm{j}}$

where $D^{\mathrm{t}}=\left(\begin{array}{lll}d_{\mathrm{i}} & d_{\mathrm{j}} & d_{\mathrm{a}}\end{array}\right)$ represent a set of damage parameters defined over the hinges.

Eqs. (3)-(5) define the damage flexibility matrix $F^{\mathrm{d}}$. Considering damage and plastic deformations, then the constitutive law is given by

$u-u^{\mathrm{p}}=u^{\mathrm{b}}+u^{\mathrm{d}}=\left[F^{\mathrm{o}}+F^{\mathrm{d}}\right] q=F(D) q$,

where $u^{\mathrm{b}}, u^{\mathrm{p}}$ and $u^{\mathrm{d}}$ represent the elastic, plastic and damage deformations, respectively, and $F^{\mathrm{o}}$ the corresponding elastic matrix.

The extension of the constitutive model for cyclic and seismic loading is direct. For this, two sets of scalar damage variables are defined in order to consider positive and negative actions [12]. The corresponding constitutive equations are given by

$u-u^{\mathrm{p}}=F\left(D^{+}\right)\langle q\rangle_{+}+F\left(D^{-}\right)\langle q\rangle_{-}$.

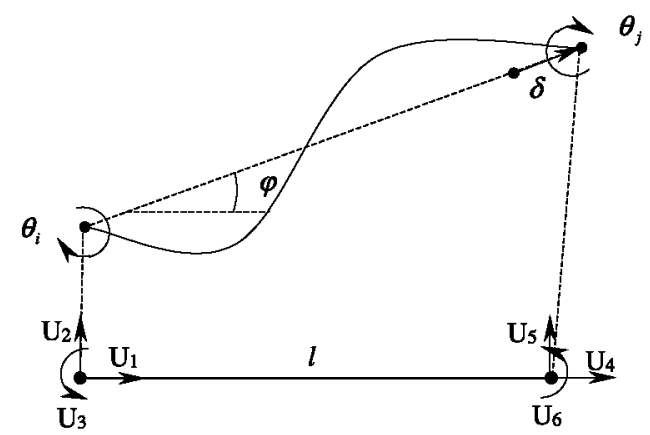

Fig. 1. Generalized deformations for the models. where $\langle q\rangle_{+}$and $\langle q\rangle_{-}$are the positive and negative parts of $q$. According to this formulation a unilateral behavior under cyclic loading is assumed.

\subsection{Thermodynamic framework}

The proposed model is derived within the framework of thermodynamics of irreversible processes. The relationship between the constitutive variables is given by a state potential. Taking as state potential the Gibbs free energy, $\chi$, it follows that

$$
\begin{aligned}
\chi= & \frac{1}{2}\langle q\rangle_{+} F\left(D^{+}\right)\langle q\rangle_{+}+\frac{1}{2}\langle q\rangle_{-} F\left(D^{-}\right)\langle q\rangle_{-} \\
& +U^{\mathrm{p}+}\left(\beta^{\mathrm{p}+}, \beta^{\mathrm{d}+}\right)+U^{\mathrm{p}-}\left(\beta^{\mathrm{p}-}, \beta^{\mathrm{d}-}\right),
\end{aligned}
$$

where $U^{p+}$ and $U^{p-}$ denote the plastic-damage potentials, function of the hardening terms $\beta^{\mathrm{p}}$ and $\beta^{\mathrm{d}}$.

From the Gibbs potential, the state laws are derived as follows:

$$
\begin{array}{ll}
Y^{+}=\frac{\partial \chi}{\partial D^{+}}, & Y^{-}=\frac{\partial \chi}{\partial D^{-}}, \\
V^{\mathrm{p}+}=\frac{\partial U^{\mathrm{p}+}}{\partial \beta^{\mathrm{p}+}}, & V^{\mathrm{p}-}=\frac{\partial U^{\mathrm{p}-}}{\partial \beta^{\mathrm{p}-}}, \\
V^{\mathrm{d}+}=\frac{\partial U^{\mathrm{d}+}}{\partial \beta^{\mathrm{d}+}}, & V^{\mathrm{d}-}=\frac{\partial U^{\mathrm{d}-}}{\partial \beta^{\mathrm{d}-}},
\end{array}
$$

where $Y^{+}$and $Y^{-}$, variables associated with $D^{+}$and $D^{-}$, correspond to the energy release rates [3] and $V^{\mathrm{p}}$ and $V^{\mathrm{d}}$ are associated with $\beta^{\mathrm{p}}$ and $\beta^{\mathrm{d}}$, respectively.

The evolution of damage and plastic variables is formulated through two dissipative potentials, $\varphi_{\mathrm{p}}$ and $\varphi_{\mathrm{d}}$, as follows:

$\mathrm{d} u^{\mathrm{p}}=\mathrm{d} \lambda^{\mathrm{p}} \frac{\partial \varphi_{\mathrm{p}}}{\partial q}, \quad \mathrm{~d} D=\mathrm{d} \lambda^{\mathrm{d}} \frac{\partial \varphi_{\mathrm{d}}}{\partial Y}$,

where $\mathrm{d} \lambda^{\mathrm{p}}$ and $\mathrm{d} \lambda^{\mathrm{d}}$ are plastic and damage consistency parameters, respectively.

\section{Modeling of low cycle fatigue for reinforced concrete}

The choice of the dissipative potentials is the critical point in the previous theory. The potentials chosen should allow modeling of phenomena such as fatigue, damage by tension, buckling, etc. Usually, in most proposed models in the literature, damage is associated only with the elastic strains through the damage energy release rate [3]. Such treatment follows the Griffith criterion and effectively assumes that damage is a function of the maximum amplitude of cyclic deformation experienced by the member, but does not depend on cumulative deformation. Current design practice is primarily concerned with the maximum single excursion of an 
element, i.e., structures are designed to sustain the maximum displacement (or force) that is expected; therefore, the proposed approach is consistent with this design philosophy.

However, failure of structures due to strong motion earthquakes may occur not only because of a single maximum excursion, but may also be caused by fatigue failure mechanisms. Because of this, the models based on the Griffith criterion presented previously are not able to represent the strength degradation that occurs due to fatigue effects. Therefore, these models are not suitable for representing this kind of failure.

To avoid these problems, several models have been proposed in the literature $[6,7]$ based on the notion of a bounding surface, which was introduced into cyclic plasticity by Dafalias and Popov [5]. For these models, in the formulation of the fatigue law, the classical concepts of yield and damage surfaces are removed and the Lubliner's concept of loading-unloading irreversibility [13] is added.

On the other hand, Ju [4] proposed a redefinition of the damage energy release rate based on consideration not only of the elastic part of the damage energy, but also of the plastic dissipation. This approach allows the characterization of damage for low cycle fatigue loading through the above energy-based damage criterion since it is linked to the history of both elastic and plastic variables.

However, using the definition of the energy release rate usually employed [3] and through the Griffith classical criterion, it is possible to take into account fatigue effects such as will be demonstrated next. For representing these effects, a suitable definition of the dissipative potentials is performed introducing a new softening term.

\subsection{Formulation of a new fatigue damage model}

Continuous fatigue damage modeling is a quite complex problem $[4,6,7]$. However, many models have been proposed in the literature [14-16] in order to quantify damage accumulation due to low cycle fatigue; most of them are based on extrapolations of Miner's rule [17] which employs a linear damage accumulation, i.e.

$D_{n_{\mathrm{i}}}=\sum \frac{n_{\mathrm{i}}}{N_{\mathrm{f}}}$

where $n_{\mathrm{i}}$ is the number of cycles for the current amplitude and $N_{f}$ is the number of cycles to failure at the same amplitude. Those models allow post-earthquake evaluation of damage, but on the whole, they have two main disadvantages: (a) consideration of the number of cycles for irregular loading histories which is not well defined and (b) quantification of the number of cycles to failure which is not well defined.
Some approaches have been proposed to convert a random loading history into an equivalent sum of cycles. Among these, cycle counting methods such as the rainflow or the range pair methods [18] are suitable for strain histories where there are only few reversals to failure.

The number of cycles to failure is often computed by a Manson-Coffin like relationship [19]:

$N_{\mathrm{f}}=C(\Delta \varepsilon)^{k}$,

where $\Delta \varepsilon$ represents the amplitude (total $\Delta \varepsilon_{\mathrm{t}}$ or plastic $\Delta \varepsilon_{\mathrm{p}}$ ) of the hysteretic loop (Fig. 2), $C$ and $k$ are materials parameters and $N_{\mathrm{f}}$ is the number of cycles to failure.

In Refs. $[16,20]$ different material parameter values for Eq. (14) were suggested, considering failure due to fatigue in the longitudinal reinforcement, which is governed mainly by flexural behavior, and failure due to transversal reinforcement fracture, which is governed mainly by shear behavior.

All previous results are based on experimental tests and a reformulation of them is needed in order to obtain a dissipative function that could be used in the model presented in Section 2.

From the Griffith dissipative potential used in Refs. [1,2] for monotonic loading, the following dissipative function is proposed here in order to model strength degradation due to low cycle fatigue:

$g=Y-\left[Y_{\mathrm{cr}}+Z(D) \xi(\omega)\right]$,

where $Z(D)$ is a hardening term as defined in Ref. [1] and $\xi(\omega)$ is a new fatigue function required to satisfy the following conditions:

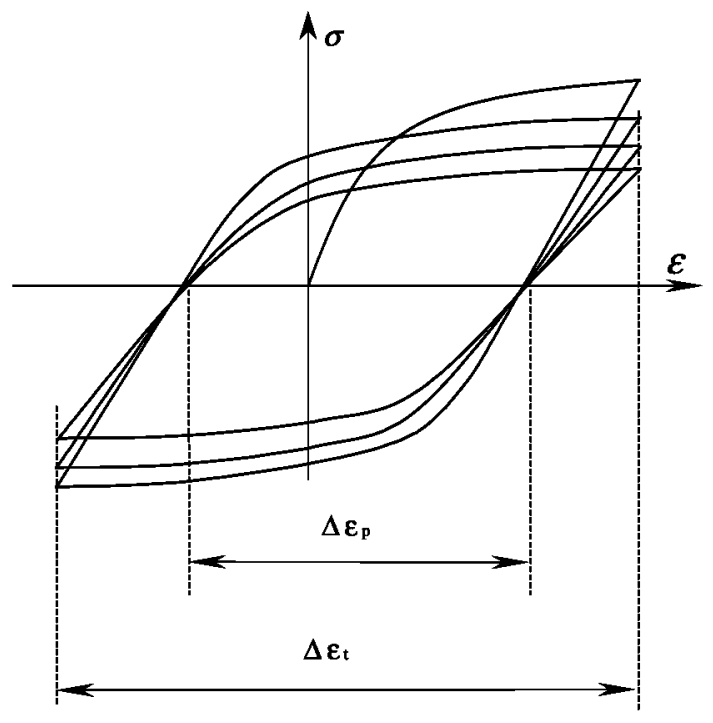

Fig. 2. Total and plastic strain amplitude. 
$\xi(\omega)=1 \Longleftrightarrow \omega \leqslant \omega_{\min }$,

$\xi(\omega)=1 \Longleftrightarrow \omega=\omega_{\max }$,

$\omega$ being a cumulative parameter which will be defined later. Physically, this model is equivalent to introduce a new term of isotropic softening related to the fatigue effects as is sometimes done in some plasticity models. With the introduction of this fatigue term, the damage domain takes the shape presented in Fig. 3.

In the same way, coupling between damage and plasticity requires the introduction of some modifications in the plastic function. The new function is written as

$f=|M-X|-\left(M_{\mathrm{y}}+R \sqrt{\xi(\omega)}\right)$.

The keypoint of this new formulation is a suitable definition of the fatigue function $\xi(\omega)$. Different evaluations have been done in order to identify the shape of this function trying to include the most important effects appearing in fatigue experimental tests. Good correlation between experimental and modeling results has been obtained with the following function:

$\xi\left(\tilde{\theta}, \theta_{\mathrm{t}}\right)=1-\left(\frac{\tilde{\theta}}{N_{\mathrm{f}}\left(\theta_{\mathrm{t}}\right) \theta_{\mathrm{t}}}\right)^{1 / \mu}$,

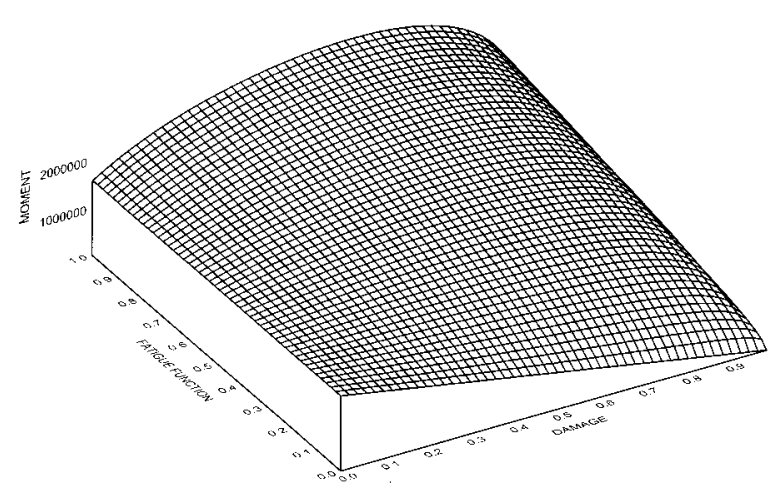

Fig. 3. Damage domain: surface $g=0$. where $\tilde{\theta}$ and $\theta_{\mathrm{t}}$ represent the total cumulative rotation and the total rotation (semiamplitude loop), respectively, and the ductility $\mu$ is defined as $\theta_{\mathrm{t}} / \theta_{\mathrm{y}} . N_{\mathrm{f}}$ being the number of cycles to failure, the term between parentheses

$\frac{\tilde{\theta}}{N_{\mathrm{f}}\left(\theta_{\mathrm{t}}\right) \theta_{\mathrm{t}}}$

can be considered as a Palmgren-Miner like relationship since the quotient $\tilde{\theta} / \theta_{\mathrm{t}}$ is the number of cycles at $\theta_{\mathrm{t}}$ semiamplitude of the loop.

Apparently, Eq. (18) introduces a new parameter in the model, the number of cycles to failure. To completely define the new model it is necessary to quantify $N_{\mathrm{f}}$. Using studies on low cycle fatigue of reinforcing steel performed by Mander et al. [20] and by Koh and Stephen [21], a relationship between the number of cycles to failure and the plastic and total deformation of the hysteretic loops, respectively, is proposed as follows:

$\varepsilon_{\mathrm{p}}=\frac{\Delta \varepsilon_{\mathrm{p}}}{2}=0.08\left(2 N_{\mathrm{f}}\right)^{-1 / 2}$

by the first authors and

$\varepsilon_{\mathrm{t}}=\frac{\Delta \varepsilon_{\mathrm{t}}}{2}=0.08\left(2 N_{\mathrm{f}}\right)^{-1 / 3}$

by the second ones. Through a suitable transformation of Eqs. (20) and (21) (Fig. 4), it is possible to obtain the following relationship between the plastic rotation and the number of cycles to failure:

$N_{\mathrm{f}}=\frac{1}{2}\left(\frac{0.16}{\theta_{\mathrm{p}}} \frac{l_{\mathrm{p}}}{d}\right)^{2}$

from the expression given by Mander et al. (Eq. 20) and

$N_{\mathrm{f}}=\frac{1}{2}\left(\frac{0.16}{d\left(\frac{M_{\mathrm{p}}}{E I}+\frac{\theta_{\mathrm{p}}}{l_{\mathrm{p}}}\right)}\right)^{3}$

from the second expression of Eq. (21). In both equations, $l_{\mathrm{p}}$ represents the plastic hinge length, and $d$ is

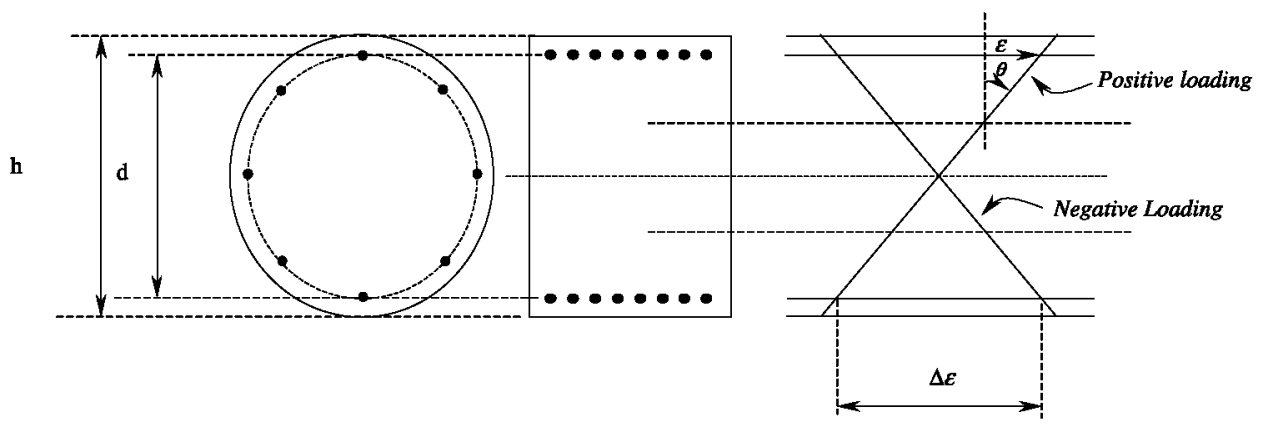

Fig. 4. Relationship between rotations and deformation. 
distance between the outer layers of steel in a rectangular section or the pitch circle diameter of the longitudinal bars in a circular section (Fig. 4). The estimation of the plastic hinge length may be done using the expression suggested by Paulay and Priesley [22] or by Eurocode 8.

Eqs. (22) and (23) have been checked with some experimental data from the literature. Fig. 5 represents the correlation between these two expressions and test results performed by Kunnath et al. [16]. To obtain these results, different columns were tested under constant amplitude reversed cyclic displacement until failure. For each constant amplitude, a value of $N_{\mathrm{f}}$ was obtained and checked with the results calculated using Eqs. (22) and (23).

In most comparisons performed, such as those from Fig. 5 , it is possible to state that the expression based on total rotations produces better correlation with experimental results than the relationship based on plastic rotations. This is probably due to the fact that the first relation takes into account more characteristics of the section, such as the plastic moment or Young's Modulus, for instance. So, Eq. (23) has been adopted in order to quantify the number of cycles to failure.

\subsection{Non-harmonic loading}

The model, as was started, can also be used without any modification for hormonic loading.

However, when non-harmonic loadings are applied, a problem appears in the model. If the loading value is changed, a jump is produced on the fatigue function such as can be observed in Fig. 6. So, an inconsistency appears when the maximum response is increased or decreased in the model.

In order to guarantee the continuity of the fatigue function (and consequently the continuity of the bending moments) the total cumulative rotation must be recalculated when the amplitude of the loops change (Fig. 7). For this, the following condition must be satisfied:

$\xi\left(\tilde{\theta}^{\text {old }}, \theta_{\mathrm{t}}^{\text {old }}\right)=\xi\left(\tilde{\theta}^{\text {new }}, \theta_{\mathrm{t}}^{\text {new }}\right)$.

From this, it can be deduced that

$\tilde{\theta}^{\text {new }}=N_{\mathrm{f}}^{\text {new }} \theta_{\mathrm{t}}^{\text {new }}\left(\frac{\tilde{\theta}^{\text {old }}}{N_{\mathrm{f}}^{\text {old }} \theta_{\mathrm{t}}^{\text {old }}}\right)^{\mu^{\text {new }} / \mu^{\text {old }}}$.

\section{Examples}

The model presented above is checked through some examples.

Fig. 8 shows the experimental [23] and numerical results of the hysteretic response of a circular crosssection column of reinforced concrete retrofitted with a steel jacket. The column was subjected to a constant axial load of $1780 \mathrm{kN}$ and lateral displacement was piloted. The unknown parameters of the model have been computed using the following characteristics for positive and negative actions: $E I / L=282.22 \mathrm{kNm}$; cracking moment is, $M_{\mathrm{cr}}^{+}=M_{\mathrm{cr}}^{-}=244 \mathrm{kN} \mathrm{m}$; plastic moment,

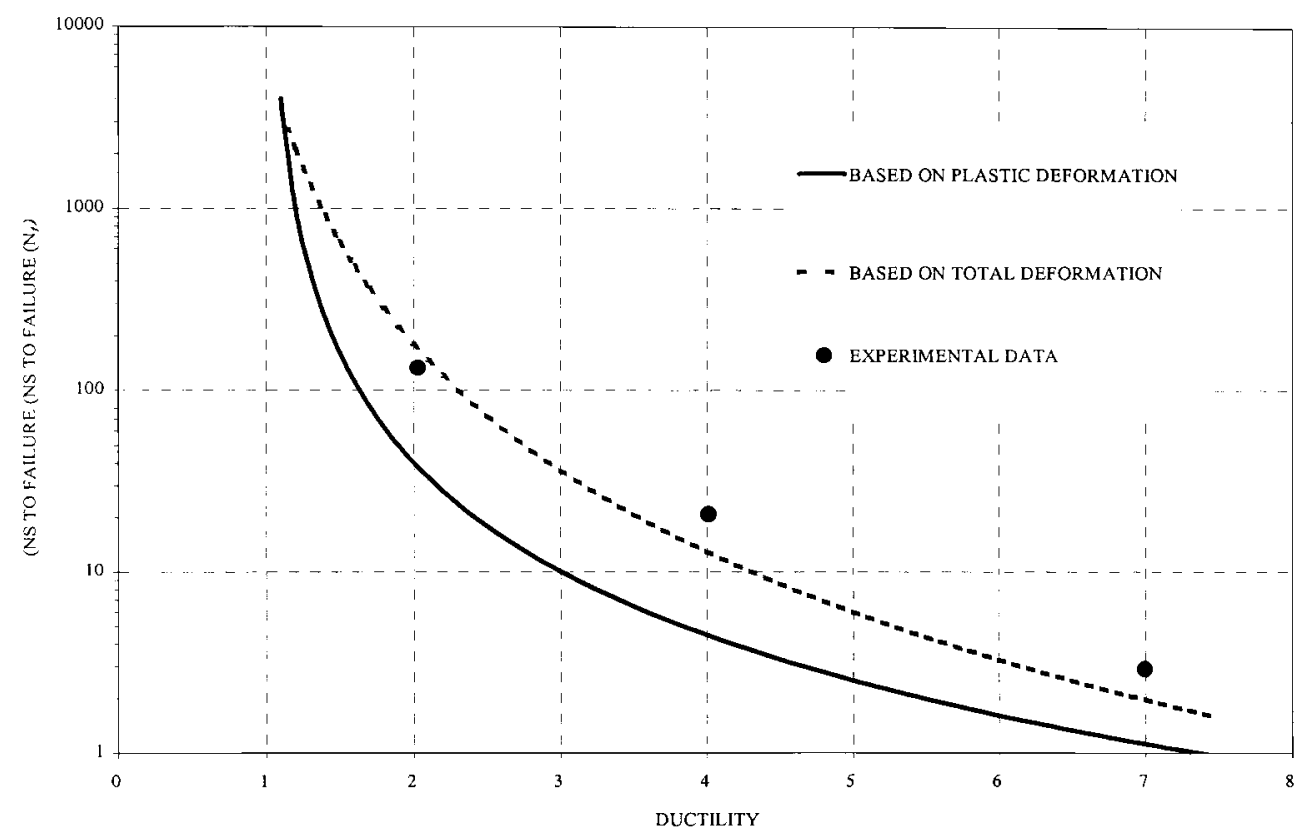

Fig. 5. Correlation between number of cycles to failure. 

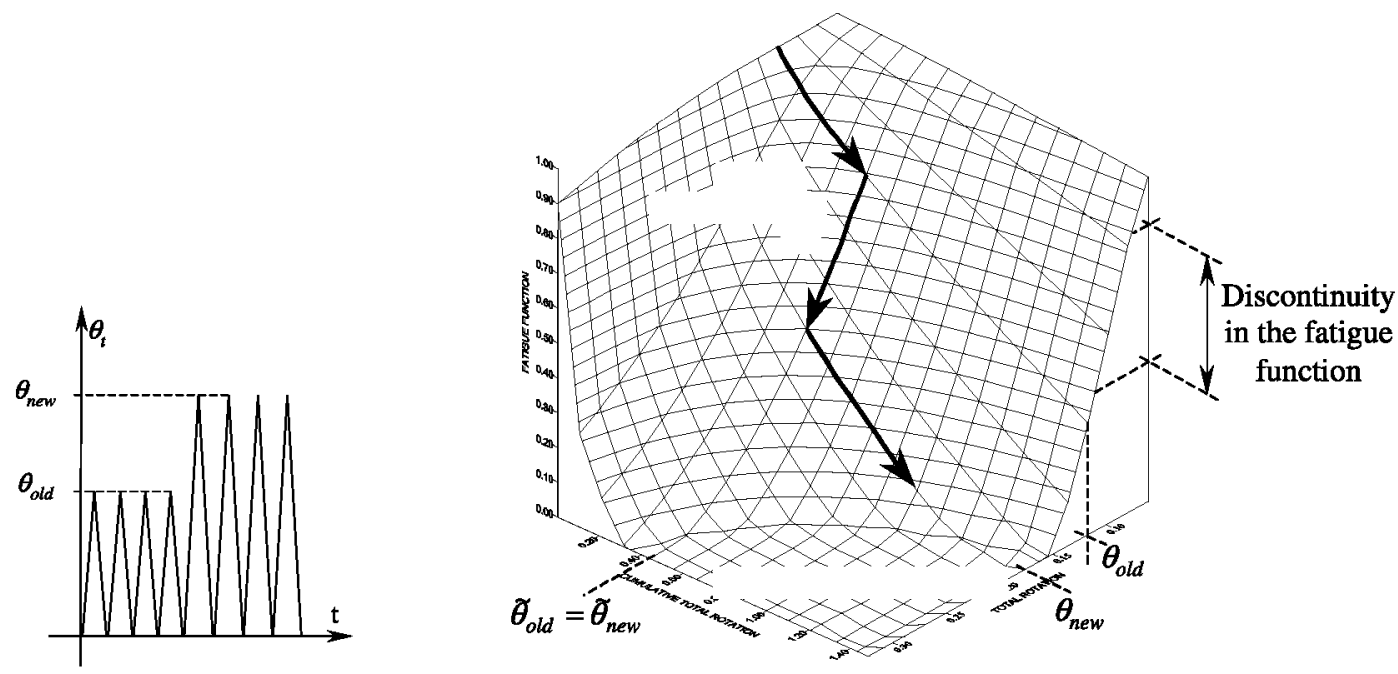

Fig. 6. Discontinuity in the fatigue function.

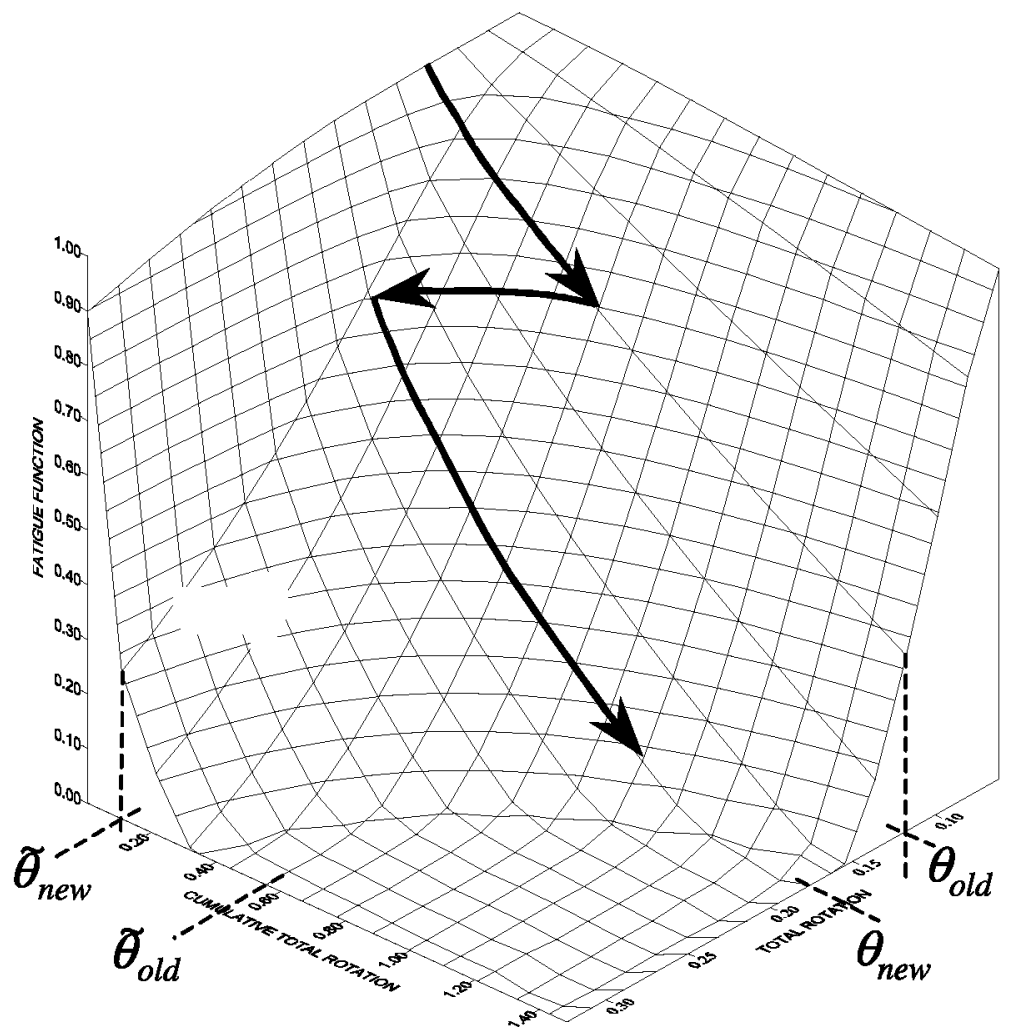

Fig. 7. Recomputation of total cumulative rotation.

$M_{\mathrm{p}}^{+}=\mathrm{M}_{\mathrm{p}}^{-}=671 \mathrm{kNm}$; ultimate moment, $M_{\mathrm{u}}^{+}=\mathrm{M}_{\mathrm{u}}^{-}=$ $1051 \mathrm{kN} \mathrm{m}$; plastic deformation at the ultimate moment, $\theta_{\mathrm{pu}}^{+}=\theta_{\mathrm{pu}}^{-}=0.03$.

These coefficients can be obtained from classic theory of $\mathrm{RC}$ when the characteristics of the member (length, area of the cross-section, amount and distribution of the reinforcement and so on) are known. In order to calculate the ultimate plastic rotation, many expressions have been suggested for the equivalent length of the plastic hinge $[22,24]$. 


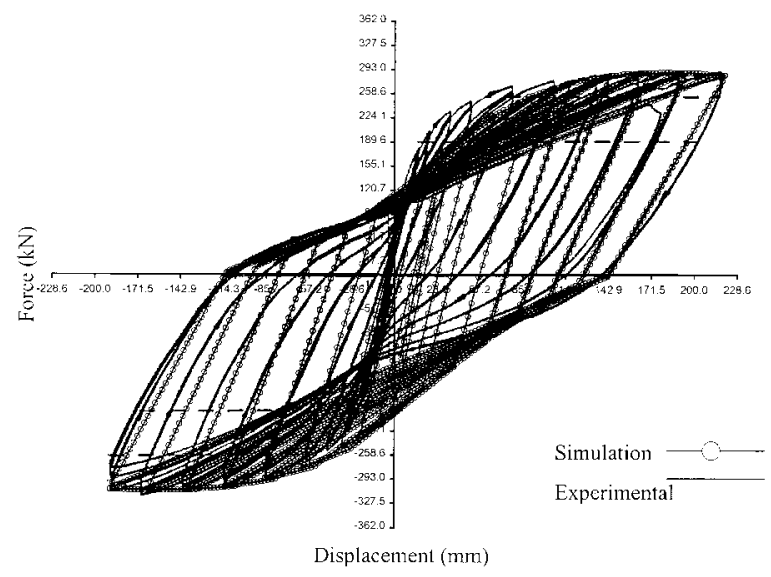

Fig. 8. Numerical and experimental results for example 1.

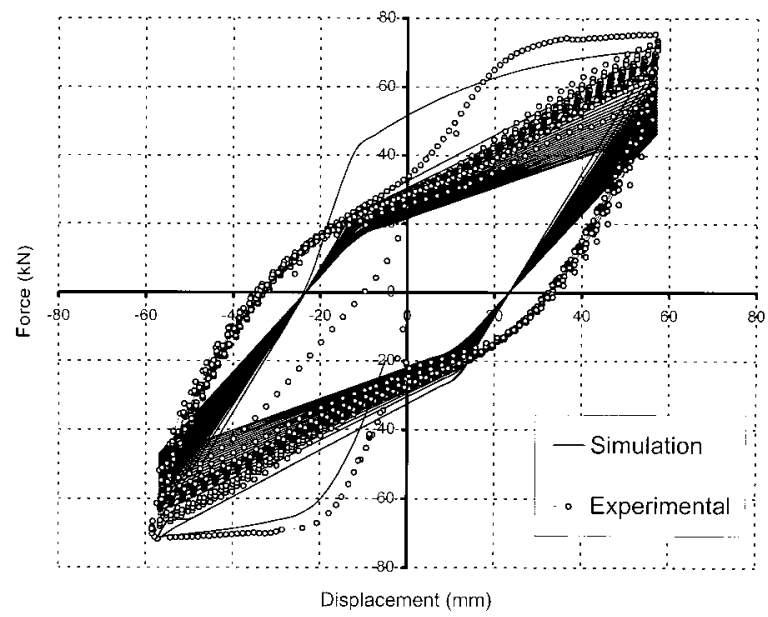

Fig. 9. Numerical and experimental results for example 2.
Obviously, the performance of the model will depend on the quality of the methods used for their calculation. To check the quality of the numerical model, in this simulation these coefficients were taken from the experimental results.

As the results show, a good agreement is reached in cases where the fatigue effects do not exist.

Fig. 9 represents experimental and numerical results using the new dissipative function. Results from Fig. 9 are referred to a circular cross-section reinforced concrete column [16] which was tested under constant amplitude cycles at $4 \%$ lateral drift. An axial load of $222 \mathrm{kN}$ was applied. For this column, necking of spirals and some bar buckling was observed at cycle 18 reaching the hoop failure at cycle 25 . The numerical simulation was performed with the following parameters obtained from the experimental tests: $E I / L=2.51 \times 10^{7} \mathrm{Nm} ; M_{\mathrm{cr}}^{+}=$ $M_{\mathrm{cr}}^{-}=27.420 \mathrm{kNm} ; M_{\mathrm{p}}^{+}=M_{\mathrm{p}}^{-}=87.808 \mathrm{kNm} ; M_{\mathrm{u}}^{+}=$ $M_{\mathrm{u}}^{-}=98.784 \mathrm{kNm} ; \theta_{\mathrm{pu}}^{+}=\theta_{\mathrm{pu}}^{-}=0.054$.

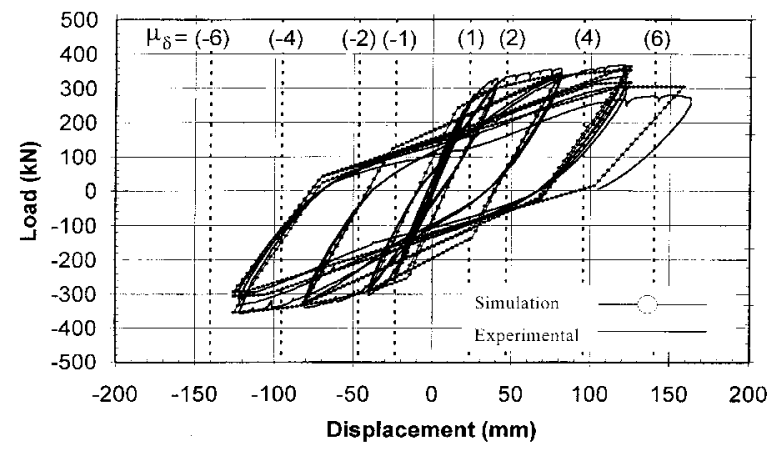

Fig. 10. Numerical and experimental results for example 3.

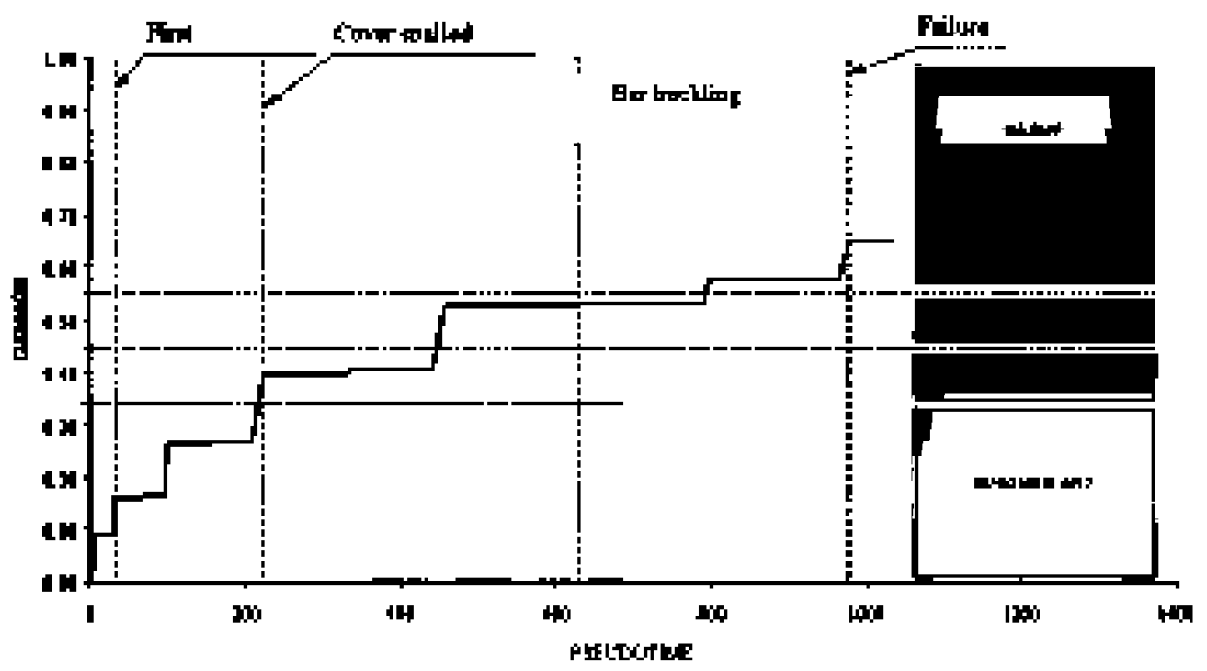

Fig. 11. Damage evolution for example 3. 
Fig. 10 shows experimental and numerical results of a rectangular cross-section reinforced concrete column with moderate confinement tested by Wehbe et al. [25].

As in the previous cases, the column was subjected to a constant axial load of $641 \mathrm{kN}$ and a lateral cyclic load history of increasing amplitude was applied. The numerical simulation has been done using the parameters: $E I / L=2.21 \times 10^{7} \mathrm{Nm} ; M_{\text {cr }}^{+}=M_{\text {cr }}^{-}=210 \mathrm{kNm} ; M_{\mathrm{p}}^{+}=$ $M_{\mathrm{p}}^{-}=643 \mathrm{kNm} ; M_{\mathrm{u}}^{+}=M_{\mathrm{u}}^{-}=850 \mathrm{kNm} ; \quad \theta_{\mathrm{pu}}^{+}=\theta_{\mathrm{pu}}^{-}=$ 0.029 . Fig. 11 shows the evolution of the damage index in the numerical simulation and includes the physical damage occurring during the test. Four levels of damage have been identified: insignificant, minor, moderate and heavy. During the test, the cover concrete started to spall at a displacement ductility of approximately $\mu_{\delta}= \pm 2$. This corresponds in the model to a damage of 0.4. The bars began to buckle for a ductility of \pm 5.2 . If the specimen was pushed to a ductility of 7 , the longitudinal bars on the compression side completely buckled and the load capacity was reduced in a high percentage. At this point, the specimen was considered to have failed which corresponds to a damage in the model of approximately 0.65. According to Fig. 11, the damage calculated could be considered heavy from a value of 0.55 .

\section{Conclusions}

A simplified model for reinforced concrete structures under hysteretic loading has been proposed. The results obtained are very promising since a good correlation was obtained between experimental and numerical results under cyclic loading. Unlike other models, it is possible to simulate the strength degradation due to low cycle fatigue effects through a suitable formulation of dissipative functions.

Further studies are needed in order to establish a relationship between the damage variable and physical damage in structural members with the purpose of establishing a good framework for decision making in the seismic retrofitting of structures. In the model presented, the damage index is associated with the cracking level of the concrete, plastic rotations are related to plastic deformations in the reinforcement and the strength degradation due to low cycle fatigue is associated with the fatigue in the longitudinal reinforcement.

The model does not take into account the pinching effect observed in response of concrete. However, the approach presented is amenable for further generalizations that are currently being studied.

\section{Acknowledgements}

The authors wish to express their gratitude to Julio Flórez-López for enlightening discussions during his sabbatical stay in Madrid supported by the Ministerio de Educación y Cultura.

\section{References}

[1] Cipollina A, López-Hinojosa A, Flórez-López J. A simplified damage mechanics approach to nonlinear analysis of frames. Comput Struct 1995;54:1113-26.

[2] Flórez-López J. Simplified model of unilateral damage for RC frames. J Struct Engng 1995;121:1765-72.

[3] Lemaitre J. A continuous damage mechanics model for ductile fracture. J Engng Mater Technol 1985;107:83-9.

[4] Ju JW. On energy-based coupled elastoplastic damage theories: constitutive modeling and computational aspects. Int J Solids Struct 1989;25:803-33.

[5] Dafalias YF, Popov EP. A model of nonlinearly hardening materials for complex loading. Acta Mech 1975;21: 173-92.

[6] Suaris W, Ouyang C, Fernando VM. Damage model for cyclic loading of concrete. J Engng Mech 1990;116:1020 35 .

[7] Marigo JJ. Modelling of brittle and fatigue damage for elastic material by growth of microvoids. Engng Fract Mech 1985;21:861-74.

[8] Lemaitre J. A Course on Damage Mechanics. Berlin: Springer, 1996.

[9] Ortiz M. A constitutive theory for the inelastic behavior of concrete. Mech Mater 1985;4:67-93.

[10] Mazars J, Berthaud Y. Une Technique expérimentale appliquée au béton pour créer un endommagement diffus et mettre en évidence le caractére uniláteral. Comte Rendu de l'Académie des Sciences 1989;308:579-84.

[11] Cohn MZ, Franchi A. Structural plasticity computer system STRUPL. J Struct Div 1979;105:789-804.

[12] Labedeze P. On a anisotropic damage theory. Proc. CNRS Int Colloquium Failure Criteria of Struct Media, Villars de Lans, France, 1983.

[13] Lubliner J. A simple theory of plasticity. Int J Solids Struct 1974;10:313-9.

[14] Suidan MT, Eubanks RA. Cumulative fatigue damage in seismic structures. J Struct Div 1973;99:923-43.

[15] Krawinkler H, Zohrei M. Cumulative damage in steel structures subjected to earthquake ground motions. Comput Struct 1983;16:531-41.

[16] Kunnath SK., El-Bahy A, Taylor A, Stone W. Cumulative seismic damage of reinforced concrete bridge piers. Technical Report NCEER-97-0006. National Center for Earthquake Engineering Research, State University of New York, Buffalo, 1997.

[17] Miner MA. Cumulative damage in fatigue. J Appl Mech 1945;12:159-64.

[18] Dowling NE. Fatigue failure predictions for complicated stress-strain histories. J Mater 1972;7:71-87.

[19] Manson SS. Behavior of materials under conditions of thermal stress. Heat Transfer Symposium, University of Michigan Engineering Research Institute, Ann Harbor, Michigan, 1953. p. 9-75.

[20] Mander JB, Panthaki FD, Kasalanati A. Low-cycle fatigue behavior of reinforcing steel. J Mater Civil Engng ASCE $1994 ; 6: 453-68$. 
[21] Koh SK, Stephen RI. Mean stress effects on low-cycle fatigue for high strength steel. Fatigue Fract Engng Mater Struct 1991;14:413-28.

[22] Paulay T, Priestley MJN. Seismic design of reinforced concrete and masonry buildings. New York: Wiley, 1992.

[23] Chai YH, Priesley MJ, Seible F. Seismic retrofit of circular bridge columns for enhanced flexural performance. ACI Struct J 1991;88:572-84.
[24] Watson S, Zahn FA, Park R. Confining reinforcement for concrete columns. J Struct Engng ASCE 1994;120(6): $1798-824$.

[25] Wehbe N, Saiidi M, Sanders D, Douglas B. Ductility of rectangular reinforced concrete bridge columns with moderate confinement. Technical Report NCEER-96-0003. National Center for Earthquake Engineering Research. State University of New York, Buffalo, 1996. 